Pacific Journal of Mathematic 


\title{
MINIMAL BASIS AND INESSENTIAL DISCRIMINANT DIVISORS FOR A CUBIC FIELD
}

\author{
LEONARD TORNHEIM
}

In terms of the coefficients $\alpha, \beta, \gamma$ of a defining equation

$$
\phi(\theta)=\theta^{3}+\alpha \theta^{2}+\beta \theta+\gamma=0
$$

of a cubic field $F$ over the rational number field $Q$, Albert [1] has given an explicit formula for a minimal basis, that is, a basis of the integers of $Q(\theta)$ over the rational integers. We solve this same problem with a shorter proof and a simpler result. This basis is then used to find the maximal inessential discriminant divisor, that is, the square root of the quotient of the g.c.d. of the discriminants of all integers of $Q(\theta)$ by the discriminant of $Q(\theta)$. It is known [3] that the only prime dividing it is 2 ; we determine the power as $2^{0}$ or $2^{1}$.

We first secure a normalized generating quantity.

Lemma 1. If $K$ is any cubic field, then $K=Q(\theta)$ with

$$
\theta^{3}+a \theta^{2}+b=0
$$

where (i) $a$ and $b$ are rational integers, (ii) no factor of a has its cube dividing $b$, and (iii) if $3 \| a$, then the discriminant $\Delta=-b\left(4 a^{3}+27 b\right)$ of $\theta$ is not divisible by $3^{4}$ unless $3 \mid b$.

Here $g^{n}|| y$ means $g^{n} \mid y$ and $g^{n+1} \nmid y$.

Proof. The substitution $\theta^{\prime}=\theta+\alpha / 3$ is used to obtain an equation of form (1) with $a$ zero. Follow this by the substitution $\theta^{\prime}=1 / \theta$ to obtain (2). For Conditions (i) and (ii) it is obvious that a substitution $\theta^{\prime}=k \theta$ will be effective. If (iii) does not hold apply the substitution $\theta^{\prime}=a b-3 b \theta+a^{2} \theta^{2}$; then $\theta^{\prime 3}+$ $c \theta^{\circ 2}+d=0$ where

$$
c=-a\left(6 b+a^{3}\right), \quad d=-b^{3}\left(4 a^{3}+27 b\right)=b^{2} \Delta .
$$

Received February 10, 1954.

Pacific J. Math. 5 (1955), 623-631 
Now $3^{2} \| c$ since $(b, 3)=1$. Also $3^{4} \mid d$. If $3^{6} \mid d$, then the quantity $\theta^{\prime \prime}=\theta^{\prime} / 9 s$ satisfies the conditions of the lemma, where $s$ is the largest integer for which $(s, 3)=1, s \mid c$, and $s^{3} \mid d$. If $3^{6} \nmid d$ use $\theta^{\prime \prime}=\theta^{\prime} / 3 s$.

Essentially the following lemma is given by Sommer [2; p. 261].

Lemma 2. The integers of $Q(\theta)$, where $\theta$ is described in Lemma 1, have a basis over the integers given by

$$
\omega_{1}=1, \quad \omega_{2}=\frac{-B+\theta}{D}, \quad \omega_{3}=\frac{B^{2}+a B+(B+a) \theta+\theta^{2}}{D^{2} D_{1}}
$$

with $B, D, D_{1}$ rational integers satisfying

$$
\begin{aligned}
3 B+a & \equiv 0(D), \\
3 B^{2}+2 a B & \equiv 0\left(D^{2} D_{1}\right), \\
B^{3}+a B^{2}+b & \equiv 0\left(D^{3} D_{1}^{2}\right), \\
-\Delta=b\left(4 a^{3}+27 b\right) & \equiv 0\left(D^{6} D_{1}^{2}\right),
\end{aligned}
$$

and $D, D_{1}$ are maximal subject to these conditions.

Proof. We shall first prove that $D=1$. Let $p$ be a prime dividing $B$ and $D$. By (3), $p$ also divides $a$. But then by $(5), p^{3} \mid b$, contradicting the choice of $\theta$. Hence $(B, D)=1$.

From (3) and (4), we have $a B \equiv 0(D)$. Therefore $D \mid a$. But by (3), $D=3$ or 1 .

If $D=3$, then $3 \nmid b$ because from ( 5$)$ we would get $D \mid B$. But then (6) contradicts (iii) of Lemma 1 . Hence $D=1$.

Therefore the problem is equivalent to determining the largest $D_{1}$ for which there is a solution $B$ satisfying (4), (5), (6), when $D=1$. It is sufficient to find solutions of these congruences with $D_{1}$ replaced by prime powers $p^{r}$ and then $D_{1}$ will be their product. A value of $B$ can be found from solutions modulo $p^{r}$ by using the Chinese remainder theorem.

Thus we wish to determine the maximal value $e$ of $r$ for which there exists a solution $B$ of the simultaneous congruences

$$
B(3 B+2 a) \equiv 0\left(p^{r}\right),
$$




$$
\begin{array}{r}
B^{3}+a B^{2}+b \equiv 0\left(p^{2 r}\right), \\
-\Delta=b\left(4 a^{3}+27 b\right) \equiv 0\left(p^{2 r}\right) .
\end{array}
$$

The power $p^{e}$ exists because of (9); in fact if $p^{u} \| \Delta$, then $e \leq s$, where $s=[u / 2]$.

Case I. $(p, 3 b)=1$. Then $e=s$. For, let $B$ be a solution of

$$
L=3 B+2 a \equiv 0\left(p^{s}\right)
$$

hence $(7)$ is satisfied. By (9)

$$
4 a^{3} \equiv-27 b\left(p^{2 s}\right)
$$

Now

$$
L^{3}-3 a L^{2} \equiv 0\left(p^{2 s}\right)
$$

This on expansion gives

$$
27 B^{3}+27 B^{2} a-4 a^{3} \equiv 0\left(p^{2 s}\right),
$$

which with the above formula shows that (8) is satisfied. Thus (7), (8), (9) hold with $r=s$. Hence $e \geq s$. But since $e \leq s$ we have $e=s$.

\section{Case II. $p \mid 3 b$.}

IIi. $(p, 2 a)=1$. Then $e=s$. For, by (9), $p^{u} \| b$. Simply take $B \equiv 0\left(p^{s}\right)$ to see that (7), (8), (9) hold with $r=s$.

IIii. $p|b, p| a$. Then $e=0$ if $p \| b$ and $e=1=s-1$ if $p^{2} \| b$. Notice that $p^{3} \nmid b$ by (ii) of Lemma 1. First, if $p^{2} \mid b$, taking $B \equiv 0(p)$ presents a solution of the congruences with $r=1$; thus $e \geq 1$. On the other hand, if $e \geq 1$, then $p \mid B$ by (8); so that $p^{2} \mid b$ again by (8). Finally, if $e>1$ then $p^{3} \mid b$ by (8) since $p \mid B$ by the preceding sentence. This is a contradiction to (ii) of Lemma 1 ; hence $e \leq 1$. It is easy to see that if $p \neq 3$, then $s=1$ when $p \| b$ and $s=2$ when $p^{2} \| b$. If $p=3$, then $s=2$ unless $p^{2} \| b, p^{2} \mid a$ and then $s=3$.

IIiii. $p=3, p \mid a, p \nmid b$. Notice that then $s=1$ by (9) and (iii) of Lemma 1 . IIiii (1). $3^{2} \mid a$. Then $e=0$ unless $b \equiv \pm 1\left(3^{2}\right)$ in which case $e=1$. Now 
$e \leq s=1$. Furthermore, the fact that $e=1$ if and only if $b \equiv \pm 1\left(3^{2}\right)$ is a consequence of (8) since only then does $B^{3}+b \equiv 0\left(3^{2}\right)$ have a solution for $(3, b)=1$, the solution being given by $B \equiv-b(3)$; (7) and (9) always hold with $r=1$.

IIiii (2). $3 \| a$. Then $e=0$, unless $b+a \equiv \pm 1\left(3^{2}\right)$, in which case $e=1$. That $e \leq 1$ is a consequence of (9) and (iii) of Lemma 1. If $r=1$, then (7) and (9) always hold and (8) has a solution if and only if $b+a \equiv \pm 1\left(3^{2}\right)$. For, if $B$ satisfies (8) then $3 \nmid B$; hence $B^{2} \equiv 1(3), a B^{2}+b \equiv a+b\left(3^{2}\right)$. But $B^{3} \equiv \pm 1\left(3^{2}\right)$ so that $a+b \equiv-B^{3} \equiv \mp 1\left(3^{2}\right)$. Conversely, if $a+b \equiv \mp 1\left(3^{2}\right)$, take $B \equiv-(a+b) \equiv \pm 1 \equiv-b(3)$; then $B^{3}+a B^{2}+b \equiv \pm 1+a+b \equiv 0\left(3^{2}\right)$.

Iliv. $p=2,2 \mid b, 2 \nmid a$. Define $t$ and $c$ by $2^{t} \| b, b=2^{t} c$.

IIiv ( 1$). t$ odd. From ( 7$), 2 \mid B$. In the expression on the left in (8), there is only one term, either $a B^{2}$ or $b$, containing 2 to the lowest power. Hence $e \leq[t / 2]$. But $B \equiv 0\left(2^{r}\right)$ with $r=[t / 2]$ does provide a solution of the three congruences. Hence $e=[t / 2]$. Notice that $e=s-1$ since $u=t+1$ if $t=1$ but $u=t+2$ if $t>1$.

IIiv (2). $t=2$. Let $4^{w} \|\left(4 a^{3}+27 b\right)$, then $w \geq 1$. Set $4 a^{3}+27 b=4^{w} H$. By (9), $e \leq w+1$. Now $e \geq w$ simply by replacing $s$ by $w$ in the solution of Case I. It remains to determine when $e=w+1$. Then from (7), $2 \mid B$ and from (8), $2^{2} \nmid B$. Also from (7), $3 B+2 a \equiv 0\left(2^{w}\right)$; that is, $3 B=-2 a+2^{w} S$. Now the product of 27 with the congruence $(8)$ gives

$$
4 a^{3}-3.2^{2 w} a S^{2}+2^{3 w} S^{3}+27 b \equiv 0\left(2^{2 w+2}\right) .
$$

Hence

$$
2^{3 w} S^{3}+2^{2 w} H-3 \cdot 2^{2 w} a S^{2} \equiv 0\left(2^{2 w+2}\right)
$$

or

$$
2^{w} S^{3}+H-3 a S^{2} \equiv 0\left(2^{2}\right)
$$

If $S \equiv 0(2)$, then $H \equiv 0(4)$, an impossibility. Hence $S$ is odd, $S^{2} \equiv 1(4)$, $S^{3} \equiv S(4)$, and

$$
2^{w} S+H+a \equiv 0\left(2^{2}\right) .
$$

But since $w \geq 1$, we have $2^{w} S \equiv 2^{w}\left(2^{2}\right)$. Hence 


$$
2^{w}+H+a \equiv 0(4) \text {. }
$$

If $w=1$, then $H \equiv a^{3}+27 c \equiv 0(2)$, a contradiction to (10). Hence $w>1$. Conversely, if $(10)$ is true, then all the congruences in this paragraph are satisfied by taking $S$ odd; that is, by taking for $B$ a solution of

$$
3 B+2 a \equiv 2^{w}\left(2^{w+1}\right) .
$$

Hence $e=w+1$ if and only if (10) is satisfied; that is, $H+a \equiv 0(4)$. Notice from the definition of $w$ that $u=2+2 w$; hence $s=w+1$.

IIiv (3). $t=2 v(v>1)$. From (9), $u=2 v+2$; hence $e \leq s=v+1$. Now $B \equiv 0\left(2^{v}\right)$ yields a solution of the congruences with $r=v$; hence $e \geq v$. We determine when $e=v+1$. Then from (7), $B$ is even. Again from (7) either $2 \| B$ or $2^{v} \mid B$. In the first case $v \leq 1$ by (8) and this is a contradiction to $v>1$; hence $B=2^{v} K$. Now (7) holds while (8) implies

$$
2^{3 v} K^{3}+a 2^{2 v} K^{2}+2^{2 v} c \equiv 0 \quad\left(2^{2 v+2}\right),
$$

which gives, since $v>1$,

$$
a K^{2}+c \equiv 0(4)
$$

Thus $K$ is odd and

$$
a+c \equiv 0(4) .
$$

Conversely, if this last congruence is satisfied and $B$ is taken as a solution of $B \equiv 2^{v}\left(2^{v+1}\right)$, then $B$ is a solution of (7), (8), and (9).

These deductions are summarized in the following theorem.

THEOREM 1. Let $\theta$ satisfy the conditions of Lemma 1. A minimal basis of $Q(\theta)$ is

$$
\omega_{1}=1, \omega_{2}=\theta, \omega_{3}=\left\{B_{2}+a B+(B+a) \theta+\theta^{2}\right\} / D
$$

where $L$ is a product of prime powers $p^{e}$ determined by the prime powers $p^{2 s}$ for which $\left(p^{2}\right)^{s} \| \Delta$ as described below and $B$ is a common solution of the congruences given below:

(1) If $(p, 3 b)=1$, then $e=s$ and $3 B+2 a \equiv 0\left(p^{e}\right)$.

(2) If $p \mid a, p \| b$, then $e=0$. Also $e=s-1$ if $p \neq 3$ and $e=s-2$ if $p=3$. 
(3) If $p \mid a, p^{2} \| b$, then $e=1$ and $B \equiv 0\left(p^{e}\right)$. Also $e=s-1$ unless $p=3$ and $p^{2} \mid a$ and then $e=s-2$.

(4) If $p \mid 3 b,(p, 2 a)=1$, then $e=s$ and $B \equiv 0\left(p^{e}\right)$.

(5) If $p=3,3 \mid a, 3 \nmid b$, then $e \leq 1=s$; and $e=s$ if and only if $b+a \equiv \pm 1$ (9) and then $B \equiv-b(3)$.

(6) If $p=2,(2, a)=1,2^{t} \| b$ and

(a) if $t$ is odd, then $e=s-1$ and $B \equiv 0\left(2^{e}\right)$;

(b) if $t=2$ then $e=s-1$ unless $H+a \equiv 0(4)$, where $H=-\Delta / 4^{s-1} b$, and then $e=s$. Also $3 B+2 a \equiv 2^{s-1}\left(2^{s}\right)$.

(c) if $t>2$ and even, then $e=s-1$ unless $a+c \equiv 0$ (4), where $c=b / 2^{t}$, and then $e=2$. Also $B \equiv 2^{s-1}\left(2^{s}\right)$.

The discriminant of $Q(\theta)$ is $\Delta / D^{2}$. It divides the discriminant $\Delta(\alpha)$ of every integer $\alpha$ of $Q(\theta)$ and hence their g.c.d. $G$. The largest inessential discriminant divisor $F$ is the square root of the quotient $G /\left(\Delta / D^{2}\right)$.

THEOREM 2. The largest inessential discriminant divisor $F$ is 1 except it is 2 in Case $6 \mathrm{~b}$ of Theorem 1 when

$$
H-3 a+2^{e-1} \equiv 0\left(2^{3}\right)
$$

and in Case 6c when

$$
a+c+2^{e-1} \equiv 0\left(2^{3}\right)
$$

Proof. The discriminant $\Delta(\alpha)$ of an integer $\alpha=c_{1} \omega_{1}+c_{2} \omega_{2}+c_{3} \omega_{3}$ can be found from the formula

$$
\Delta(\alpha)=\left|a_{i j}\right|^{2} \Delta(\theta)
$$

where the elements of the determinant $\left|a_{i j}\right|=\left|a_{i j}(\alpha)\right|$ are defined by

$$
\alpha^{i-1}=a_{i 1}+a_{i 2} \theta+a_{i 3} \theta^{2} \quad(i=1,2,3),
$$

Since the discriminant of $\alpha$ is unaltered by addition of a rational number, we have

$$
\Delta(\alpha)=\Delta\left(c_{2} \omega_{2}+c_{3} \omega_{3}\right)=\Delta(\beta),
$$

where 


$$
\beta=\left[c_{2}+c_{3}(B+a) / D\right] \theta+\left(c_{3} / D\right) \theta^{2} .
$$

In computing $\beta^{2}$ use the fact that $\theta^{3}=-a \theta^{2}-b$ and $\theta^{4}=a^{2} \theta-b \theta+a b$. Also since the first row of $\left|a_{i j}(\beta)\right|$ is $1,0,0$, any rational terms can be ignored. Hence,

$$
\left|a_{i j}\right|=\frac{c_{3}^{3}\left(B^{3}+a B^{2}+b\right)}{D^{3}}+\frac{c_{2} c_{3}^{2}\left(3 B^{2}+2 a B\right)}{D^{2}}+\frac{c_{2}^{2} c_{3}(3 B+a)}{D}+c_{2}^{3} .
$$

Thus

$$
\left|a_{i j}\left(\omega_{3}\right)\right|=\frac{\left(B^{3}+a B^{2}+b\right)}{D^{3}}
$$

and

$$
\left|a_{i j}\left(\omega_{2}+\omega_{3}\right)\right|-\left|a_{i j}\left(\omega_{3}\right)\right|=\frac{\left(3 B^{2}+2 a B\right)}{D^{2}}+\frac{(3 B+a)}{D}+1 .
$$

Now, since $G D^{2} / \Delta$ is the quotient of the g.c.d $G$ of $\left|a_{i j}\right|^{2} \Delta$ by $\Delta / D^{2}$, it equals the g.c.d of $\left|a_{i j}\right|^{2} D^{2}$. Hence the inessential discriminant divisor $F$ is the g.c.d of $\left|a_{i j}\right| D$.

To find $F$ we determine for each prime $p$ the highest power $p^{f}$ which remains in all the denominators of the $\left|a_{i j}(\alpha)\right|$ expressed in their lowest terms. Then $F$ is the quotient of $D$ divided by the product of these prime powers and thus $F$ is the product of all $p^{e-f}$.

In all cases of Theorem 1 except in 5 when $a+b \equiv \pm 1\left(3^{2}\right)$, in $6 \mathrm{~b}$ when $H+a \equiv 0\left(2^{2}\right)$, and in $6 \mathrm{c}$ when $a+c \equiv 0\left(2^{2}\right), B$ may be chosen to satisfy either

$$
B \equiv 0\left(p^{3 e}\right)
$$

or

$$
3 B+2 a \equiv 0\left(p^{2 e}\right) .
$$

In these cases (15) implies, since its first term is then integral, that $e=f$ when $p \nmid a$. But if $p \mid a$ then $p \mid b$ and since we need consider only $e>0$ we have Case 3 of Theorem 1 . Then (14) with $B \equiv 0\left(p^{3 e}\right)$ shows that $f=1=e$. 
Next, in Case 5 when $b+a \equiv \pm 1\left(3^{2}\right), 3 \nmid B$. If $3^{2} \mid a$, then (15) implies that $f=1=e$. But if $3 \| a$ then $a=3 a_{1}$ and $a_{1}^{3}+b \neq 0$ (3) by (iii) of Lemma 1 . Were $f=0$, then $B+2 a_{1} \equiv 0(3)$ by (15), which implies $B \equiv a_{1}$ (3). But then

$$
B^{3}+a B^{2}+b \equiv a_{1}^{3}+b \not \equiv 0(3),
$$

a contradiction to (8). Hence again $f=e$.

In both Cases $6 \mathrm{~b}$ and $6 \mathrm{c}, 2 \mid B$ by ( 7$)$. Now

$$
\left|a_{i j}\left(\omega_{2}+\omega_{3}\right)\right|+\left|a_{i j}\left(-\omega_{2}+\omega_{3}\right)\right|-2\left|a_{i j}\left(\omega_{3}\right)\right|=\frac{2(3 B+a)}{D} .
$$

Since $2 \| 2(3 B+a)$, we have $f \geq e-1$.

We now consider in particular Case $6 \mathrm{~b}$ when $H+a \equiv 0(4)$. Then $3 B=$ $-2 a+2^{e-1} Q$, where $Q$ is odd. Thus

$$
27\left(B^{3}+a B^{2}+b\right)=4 a^{3}+27 b-3 Q^{2} a 2^{2 e-2}+Q^{3} 2^{3 e-3} .
$$

Hence if $f=e-1$, then

$$
H-3 a+2^{e-1} \equiv 0\left(2^{3}\right)
$$

by (14), and if this is satisfied then $f=e-1$. For, the first term in (13) has numerator divisible by $2^{2 e+1}$, and $2^{e} \|\left(3 B^{2}+2 a B\right)$ and $2^{0} \|(3 B+a)$ so that

$$
2^{e+1} \mid\left[c_{2} c_{3}^{2}\left(3 B^{2}+2 a B\right)+D c_{2}^{2} c_{3}(3 B+a)\right] \text {. }
$$

Hence in lowest terms $\left|a_{i j}\right|$ has a denominator divisible by no power of $p$ greater than $e-1$.

We finally discuss Case $6 c$ when $a+c \equiv 0(4)$. Then $B=2^{e-1}+C 2^{e}$, where we may assume that $2^{e+2} \mid C$, and $b=2^{2(e-1)} c$. Hence

$$
B^{3}+a B^{2}+b \equiv 2^{3 e-3}+2^{2 e-2}(a+c)\left(2^{3 e}\right) .
$$

If $f=e-1$, then by (14) this expression must be $\equiv 0\left(2^{2 e+1}\right)$, so that

$$
2^{e-1}+a+c \equiv 0(8)
$$

If this is satisfied then $f=e-1$ because the first term of (13) has numerator divisible by $2^{2 e+1}$, and $2^{e} \|\left(3 B^{2}+2 a B\right)$ and $2^{0} \|(3 B+a)$ so that 


$$
\begin{gathered}
2^{e+1} \mid\left[c_{2} c_{3}^{2}\left(3 B^{2}+2 a B\right)+D c_{2}^{2} c_{3}(3 B+a)\right] . \\
\text { REFERENCES }
\end{gathered}
$$

1. A. A. Albert, A determination of the integers of all cubic fields, Ann. of Math., 31 (1930), $550-566$.

2. J. Sommer, Vorlesungen über Zahlentheorie, Berlin, 1907.

3. E.v. Zylinski, Zur Theorie der ausserwesentliche Diskrminantenteiler algebraischer Körper, Math. Ann. 73 (1913), $273-274$.

UNIVERSITY OF MICHIGAN 



\section{PACIFIC JOURNAL OF MATHEMATICS}

\section{EDITORS}

\section{H.L. ROY DEN}

Stanford University

Stanford, California

E. HEWITT

University of Washington

Seattle 5, Washington

\section{R. P. DILWORTH}

California Institute of Technology

Pasadena 4, California

* Alfred Horn

University of California

Los Angeles 24, California

\section{ASSOCIATE EDITORS}

\begin{abstract}
H. BUSEMANN
HERBERT FEDERER

MARSHALL HALL
\end{abstract}

\section{P.R. HALMOS}

HEINZ HOPF

ALFRED HORN
R.D. JAMES

BØRGE JESSEN

PAUL LÉVY
GEORGE PÓLYA

J.J. STOKER

KOSAKU YOSIDA
UNIVERSITY OF BRITISH COLUMBIA CALIFORNIA INSTITUTE OF TECHNOLOGY UNIVERSITY OF CALIFORNIA, BER KELEY UNIVERSITY OF CALIFORNIA, DAVIS UNIVERSITY OF CALIFORNIA, LOS ANGELES UNIVERSITY OF CALIFORNIA, SANTA BARBARA MONTANA STATE UNIVERSITY

UNIVERSITY OF NEVADA

OREGON STATE COLLEGE

UNIVERSITY OF OREGON
UNIVERSITY OF SOUTHERN CALIFORNIA STANFORD UNIVERSITY UNIVERSITY OF UTAH WASHINGTON STATE COLLEGE UNIVERSITY OF WASHINGTON

AMERICAN MATHEMATICAL SOCIETY HUGHES AIRCRAFT COMPANY SHELL DEVELOPMENT COMPANY

Mathematical papers intended for publication in the Pacific Journal of Mathematics should be typewritten (double spaced), and the author should keep a complete copy. Manuscripts may be sent to any of the editors. Manuscripts intended for the outgoing editors should be sent to their successors. All other communications to the editors should be addressed to the managing editor, Alfred Horn, at the University of California Los Angeles 24, California.

50 reprints of each article are furnished free of charge; additional copies may be obtained at cost in multiples of 50 .

The Pacific Journal of Mathematics is published quarterly, in March, June, September, and December. The price per volume (4 numbers) is $\$ 12.00$; single issues, $\$ 3.50$; back numbers (Volumes $1,2,3$ ) are available at $\$ 2.50$ per copy. Special price to individual faculty members of supporting institutions and to individual members of the American Mathematical Society: $\$ 4.00$ per volume; single issues, $\$ 1.25$.

Subscriptions, orders for back numbers, and changes of address should be sent to the publishers, University of California Press, Berkeley 4, California.

Printed at Ann Arbor, Michigan. Entered as second class matter at the Post Office, Berkeley, California.

* During the absence of E.G. Straus.

UNIVERSITY OF CALIFORNIA PRESS - BERKELEY AND LOS ANGELES 


\section{Pacific Journal of Mathematics}

\section{Vol. 5, No. $4 \quad$ December, 1955}

Richard Horace Battin, Note on the "Evaluation of an integral occurring in servomechanism theory" ............................. 481

Frank Herbert Brownell, III, An extension of Weyl's asymptotic law for eigenvalues................................. 483

Wilbur Eugene Deskins, On the homomorphisms of an algebra onto Frobenius algebras .................................. 501

James Michael Gardner Fell, The measure ring for a cube of arbitrary dimension ....................................... 513

Harley M. Flanders, The norm function of an algebraic field extension. II ............................................ 519

Dieter Gaier, On the change of index for summable series ............ 529

Marshall Hall and Lowell J. Paige, Complete mappings of finite groups . . . . 541

Moses Richardson, Relativization and extension of solutions of irreflexive relations..................................... 551

Peter Scherk, An inequality for sets of integers .................. 585

W. R. Scott, On infinite groups ........................... 589

A. Seidenberg, On homogeneous linear differential equations with arbitrary constant coefficients ......................................... 599

Victor Lenard Shapiro, Cantor-type uniqueness of multiple trigonometric integrals...

Leonard Tornheim, Minimal basis and inessential discriminant divisors for a cubic field...

Helmut Wielandt, On eigenvalues of sums of normal matrices ... 